\title{
AVALIAÇÃO DOS TRANSPLANTADOS HEPÁTICOS EM SANTA CATARINA, DE AGOSTO DE 2002 A JULHO DE 2004: RELATO DOS PRIMEIROS 25 CASOS DE UM PROCEDIMENTO INÉDITO NO ESTADO
}

\author{
Evaluation of liver transplantation recipients in Santa Catarina state from August, 2002, \\ until July, 2004: First twenty-five cases of a new procedure in our state
}

Marcelo Augusto Scheidemantel Nogara, Júlio César Wiederkher¹, Mauro Rafael da Igreja1, Jaqueline Akemi Okada, Anny Bellanda Mazzei ${ }^{2}$, Jaciara Raiter ${ }^{2}$

\begin{abstract}
RESUMO
Objetivo: A nalisar os resultados da série inicial de transplantes hepáticos no estado de Santa Catarina, realizados no Hospital Santa I sabel, município de Blumenau. Métodos: foram executados 25 transplantes em 24 pacientes portadores de insuficiência hepática terminal de variadas etiologias, no período de agosto de 2002 a jul ho de 2004. Estudo transversal e quantitativo, feito através da pesquisa de dados de prontuários médicos da data do transplante hepático. Também consideramos as internações no pós-transplante, desde que ocorridas até a data da coleta dos dados (setembro a outubro de 2005). Resultados a média de idade dos transplantados foi de 47,24 anos, sendo o sexo masculino predominante (72\%, n: 18). A sobrevida dos pacientes em três meses foi de $87,5 \%$, e a de um ano, $71 \%$. A etiologia predominante foi hepatite C concomitante a alcoolismo (24\%) e hepatite C isoladamente (24\%). A complicação mais comum no pós-operatório foi infecção ( $\mathrm{n}$ : 17). A penas um retransplante foi realizado, por trombose de artéria hepática. Condusão: Este trabal ho demonstra que o transplante hepático pode ser realizado fora dos grandes centros, beneficiando assim uma população que antes não dispunha deste serviço, e com uma tendência a ter resultados semel hantes aos serviços de ponta que realizam este procedimento há mais tempo.
\end{abstract}

Descritores: Transplante de Fígado; Insuficiência Hepática; Hepatite Crônica; Cirrose Hepática; Sepse.

Instituição:

1. Serviço de Gastroenterologia, Hepatologia e Cirurgia Geral - Equipe de Transplantes do Hospital Santa Isabel.

2. Curso de Medicina da Fundação Universidade Regional de Blumenau (FURB) Blumenau - SC

Correspondência:

Dr. Marcelo Augusto Scheidemantel Nogara

Hospital Santa Isabel - Rua Floriano Peixoto, 300, sala 201

CEP: 89010-906 - Blumenau - SC - Brasil

Tel./Fax: 5547 3322-1205

E-mail:kmnogara@terra.com.br

Recebido em: 22.02.2006

Aceito em: 30.03 .2006

\section{INTRODUÇÃO}

O primeiro transplante hepático em humanos foi realizado por Starzl, em 1963. D esde então, os resultados têm mel horado gradativamente, devido ao uso das modernas drogas imunossupressoras, mel horia na disponibilidade de doadores, controle da rejeição e das infecções, e técnicas cirúrgicas avançadas ${ }^{1}$.

Desse modo, hoje o transplante de fígado tem revolucionado o tratamento dos portadores de doença hepática terminal, sendo indicado tanto na insuficiência hepática aguda quanto na crônica, de variadas etiologias. ${ }^{2}$ Torna-se alternativa em adultos e crianças com hepatopatia grave e irreversível, na ausência de contra-indicações e, quando não há mais viabilidade de outros tipos de tratamento, clínicos ou cirúrgicos. ${ }^{3-6}$

A maioria dos pacientes com doença hepática em fase terminal apresenta cirrose, principal razão para o transplante de fígado na população adulta. Em estudo com pacientes hepatopatas, a sobrevida em cinco anos foi de $70 \%$ na apresentação da doença, e caiu para $57 \%$ a partir do momento da instalação de cirrose. ${ }^{3}$ Por isso, o transplante hepático é hoje aceito como terapêutica viável para melhorar a sobrevida e a qualidade de vida desses pacientes críticos.

Esse estudo é um relato dos transplantes hepáticos realizados no 
estado de Santa Catarina, desde o início do programa (em agosto de 2002), até julho de 2004.

\section{PACIENTES E MÉTODOS}

Os pacientes foram avaliados por uma equipe multidisciplinar constituída por: hepatologista, cirurgião, psiquiatra, enfermeira, assistente social, nutricionista e fisioterapeuta, entre outros. Essa avaliação visou basicamente o diagnóstico da hepatopatia e suas complicações. 0 diagnóstico de insuficiência hepática foi elaborado através de critérios clínicos (encefalopatia e/ou ascite; peritonite bacteriana espontânea recorrente; sangramento digestivo recidivante por hipertensão portal; fadiga ou desnutrição acentuadas; prurido intratável; síndrome hepatorrenal ou hepatopulmonar; doença óssea; colangites de repetição; neuropatia) e laboratoriais (níveis de bilirrubinas, albumina sérica e tempo de protrombina - TAP), estando os critérios laboratoriais de acordo com a classificação de Child-Pugh. ${ }^{7}$ A gravidade da doença foi caracterizada pelo escore de Child-Pugh, ${ }^{7}$ e o escore de $\mathrm{M}$ eld..$^{8}$ Os pacientes foram qualificados como Child A quando possuíam doença hepática compensada, Child $\mathrm{B}$ com dano hepático funcional significativo, e $\mathrm{C}$ hild $\mathrm{C}$ na presença de doença hepática descompensada. 0 escore do Meld maior ou igual a 15 acusa uma hepatopatia grave.

Trata-se de um estudo transversal e retrospectivo, cujo único critério de inclusão foi que o paciente submetido ao transplante já tivesse completado no mínimo um ano de pós-operatório. Por isso, foram excluídos os transplantes realizados após jul ho de 2004.

0 estudo foi feito através de coleta de dados dos prontuários dos doentes durante a permanência hospitalar por motivo de transplante. Também foram revisadas as internações posteriores, ocorridas atéa data da coleta dos dados (agosto a setembro de 2005). 0 objetivo foi buscar dados epidemiológicos (idade, sexo, procedência, etiologia dos transplantes); complicações pós-transplante e evolução desses transplantados (tempo de internação, sobrevida, ocorrência ou não de retransplante).

Os enxertos hepáticos foram captados de doadores cadáveres. Os doadores eram todos procedentes do estado de Santa Catarina, a média das idades era de 37 anos, estando três deles (12\%) com até 19 anos, seis (24\%) entre 20-29 anos, três (12\%) entre 30-39 anos, sete (28\%) entre 40-49 anos e seis (24\%), entre 50-59 anos, sendo 14,7 0 desvio padrão. A causa da morte dos doadores mais encontrada foi traumatismo crânio-encefálico, com dez casos (40\%), seguido por acidente vascular cerebral hemorrágico, com sete casos (28\%), aneurisma cerebral, três casos $(12 \%)$, acidente vascular cerebral isquêmico, dois casos (8\%), hemorragia subaracnóidea, dois casos $(8 \%)$ e encefalopatia anóxica, um caso (4\%).

Os receptores e doadores deveriam apresentar compatibilidade sangüínea $A B O$, além de peso e altura semelhantes. Exigências preenchidas, os receptores eram selecionados para receber os órgãos, de acordo com o tempo de inscrição na lista de espera.

A pós a captação, era realizado o preparo do enxerto hepático, utilizando-se as veias cavas, veia porta, artéria hepática e ductos biliares comuns do doador para as anastomoses vasculares e biliares do enxerto. Na cirurgia dos receptores, o enxerto hepático era implantado em posição ortotópica, no hipocôndrio direito, com anastomoses término-terminais entre os vasos hepáticos e os ductos biliares comuns do doador com os do receptor.
0 tempo de isquemia do enxerto variou entre quatro e 13 horas, com média de 8,7 horas e desvio padrão de 2,3. A duração da cirurgia teve uma média de 5,8 horas, variando de três horas e 15 minutos a oito horas, com desvio padrão de 1,2.

A terapia imunossupressiva preconizada foi iniciada no pósoperatório imediato com tacrolimus $5 \mathrm{mg}$ endovenoso 12/12h ou via oral quando tolerado, associado a prednisona ou metilprednisolona $500 \mathrm{mg}$ endovenoso ao dia. 0 nível sérico de tacrolimus almejado é de $12-15 \mathrm{ng} / \mathrm{ml}$ do $1^{0}$ ao 6 o pós-operatório. A pós este período foi modificado o nível sérico para 8-10 ng/ml até completar um ano da data do transplante, mantendo na seqüência a dose de 5-8ng/dl. A corticoterapia no primeiro pós-operatório foi usada na dose de $200 \mathrm{mg}$ endovenoso de $12 / 12 \mathrm{~h}$, modificando para $150 \mathrm{mg}$ endovenoso $12 / 12 \mathrm{~h}$ no segundo pós-operatório e, a partir daí, com queda de $25 \mathrm{mg}$ por dia, até chegar no sexto dia com $50 \mathrm{mg}$ endovenoso 12/12h. No sétimo pós-operatório, a dose é ajustada para 40mg endovenoso 12/12h, e são retirados $5 \mathrm{mg}$ por dia da dose até o $10^{\circ}$ dia, que permanece da mesma maneira até o 14임. Do 15ำ ao 24ํㅜ pós-operatório mantém $20 \mathrm{mg}$ endovenoso 12/12h. Do 25을 dia até completar dois meses da data do transplante é usado $15 \mathrm{mg}$ endovenoso 12/12h e, após isso, até o quarto mês é al terado para $20 \mathrm{mg}$ ao dia em dose única, caindo para $15 \mathrm{mg}$ ao dia de quatro a seis meses de pós-operatório, encerrando a imunossupressão com o uso de corticóide.

Todos os pacientes estudados receberam transfusão de hemoderivados durante o período de internamento para 0 transplante hepático.

0 diagnóstico das complicações pós-transplante foi feito através de exames de imagem (US, TC, RM , RX), endoscopia digestiva, biópsia hepática e investigação laboratorial (hemoculturas, hemograma, bioquímica renal e hepática, glicemia, entre outros).

\section{RESULTADOS}

Deagosto de 2002 a jul ho de 2004 foram realizados 25 transplantes em nossa instituição. A grande maioria dos transplantados $(96 \%, n$ $=24)$ foi catalogada como Child-Pugh B $(48 \%, n=12)$ ou C $(48 \%$, $n=12)$, sendo apenas $4 \%(n=1)$ deles classificados como Child A . Na avaliação do M eld, oito pacientes (32\%) foram classificados como Meld menor que 15 , e 17 pacientes (68\%) apresentavam Meld maior ou igual a 15 . O sexo masculino foi predominante, representando $72 \%$ dos casos $(n=18)$. A idade variou de 20 a 69 anos, com média de 48,2 anos, e desvio padrão de 12,6 , sendo $16 \%$ $(n=4)$ com $20-29$ anos, $4 \%(n=1)$ com $30-39$ anos, 32\% ( $n=$ 8) com 40 - 49 anos, $28 \%(n=7)$ com 50 - 59 anos e $20 \%(n=5)$ com 60 anos ou mais. Q uanto à procedência, seis pacientes eram de Blumenau (24\%), 17 (68\%) vinham de outras cidades do estado de Santa Catarina, e $8 \%(n=2)$ residiam em outros estados.

Houve $29 \%$ ( $n=7$ ) deóbitos no período de acompanhamento, sendo a sepse com $57,1 \%(n=4)$, a causa majoritária, seguida com um caso $(14,2 \%)$ de complicações biliares, um caso $(14,2 \%)$ de edema agudo de pulmão, e mais um caso $(14,2 \%)$ de não funcionamento primário. Com isso, a sobrevida em três meses de pós-operatório resultou $87,5 \%(n=21)$ e a de um ano ficou em $71 \%(n=17)$. (Gráfico 1). Retransplante foi realizado em apenas um paciente (4\%), devido à trombose da artéria hepática, em quatro meses após o transplante primário. 
A s três principais etiologias da injúria hepática em nossa instituição foram as seguintes: $24 \%(n=6)$ por hepatite C crônica; igualmente $24 \%$ $(\mathrm{n}=6)$ por hepatite $\mathrm{C}$ crônica concomitante a alcoolismo, seguido pela hepatopatia alcoólica isolada, com 20\% de prevalência ( $n=5)$.

0 tempo de internação hospitalar total variou de um a 42 dias, com média de 17,9 dias, e desvio padrão de 7,6. Já a permanência em terapia intensiva variou de um a 11 dias, com média de 4,5 dias e desvio padrão de 2,2. (Tabela 1).

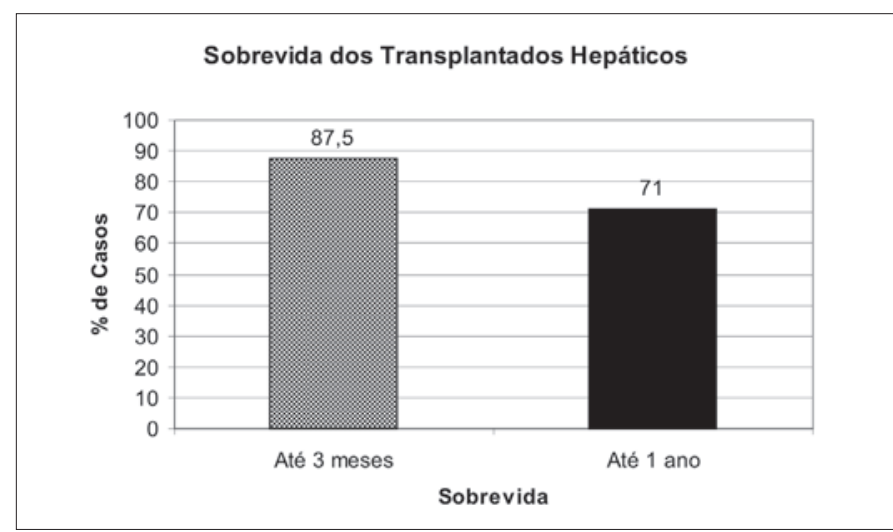

Gráfico 1. Sobrevida dos transplantes hepáticos

\begin{tabular}{|c|c|}
\hline Variáveis Analisadas & Dados Obtidos \\
\hline Número de transplantes & 25 \\
\hline \multirow[t]{3}{*}{ Child-Pugh } & A: $1(4 \%)$ \\
\hline & B: $12(48 \%)$ \\
\hline & C: $12(48 \%)$ \\
\hline \multirow[t]{2}{*}{ MELD } & $<15: 8(32 \%)$ \\
\hline & $\geq 15: 17(68 \%)$ \\
\hline \multirow[t]{2}{*}{ Sexo } & Masculino: $18(72 \%)$ \\
\hline & Feminino: 7 (28\%) \\
\hline Idade & Média: 48,2 \\
\hline \multirow[t]{3}{*}{ Procedência } & Blumenau: $6(24 \%)$ \\
\hline & Outras regiões de Santa Catarina: 17 (68\%) \\
\hline & Outros estados: 2 (8\%) \\
\hline \multirow[t]{4}{*}{ Causas de óbito } & Complicações biliares: 1 (14,2\%) \\
\hline & Edema agudo de pulmão: $1(14,2 \%)$ \\
\hline & Sepse: $4(57,1 \%)$ \\
\hline & Não funcionamento primário: 1 (14,2\%) \\
\hline \multirow[t]{2}{*}{ Sobrevida } & Três meses: 21 (87,5\%) \\
\hline & Um ano: $17(71 \%)$ \\
\hline Retransplante & Trombose da artéria hepática: 1 (4\%) \\
\hline \multirow[t]{5}{*}{ Etiologia da insuficiência hepática } & Hepatite C crônica: 6 (24\%) \\
\hline & Hepatite C crônica + álcool: 6 (24\%) \\
\hline & Álcool: 5 (20\%) \\
\hline & Hepatite auto-imune: 3 (12\%) \\
\hline & Outros: $5(20 \%)$ \\
\hline \multirow[t]{2}{*}{ Tempo de internação } & Hospital (média): 17,9 dias \\
\hline & UTI (média): 4,5 dias \\
\hline
\end{tabular}

Tabela 1. Resultado dos transplantes hepáticos em Santa Catarina
$\mathrm{Na}$ análise das complicações, observou-se que alguns pacientes apresentaram mais de uma. Como mais prevalentes apareceram as infecções, com 17 casos (um caso de erisipela, um de peritonite bacteriana espontânea, um de peritonite, cinco de sepse, um de abscesso hepático, dois de infecção do trato urinário, um de infecção de sítio operatório, dois de citomegalovirose, um de gastrenterite aguda, um de supuração de parede, e final mente, um caso de varicela). Em segundo lugar, surgiram as complicações pulmonares, com 11 casos, seguidas dos distúrbios do trato gastrintestinal, que ocorreram em dez pacientes (G ráfico 2).

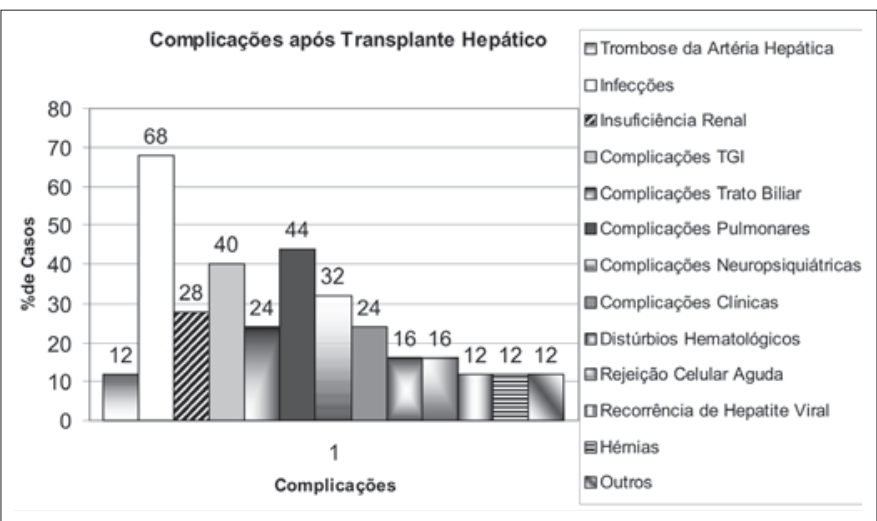

Gráfico 2. Complicações do pós-transplante

\section{DISCUSSÃO}

O perfil do transplantado de nossa instituição ficou, portanto, assim estabelecido:

0 sexo predominante foi o masculino, com $72 \%(n=18)$. Provável explicação é o fato de os homens costumarem ser mais promíscuos sexual mente, utilizarem drogas injetáveis em mai or quantidade, além de geralmente exibirem uma taxa maior de etilismo, em relação às mulheres. Com isso, estão mais expostos às hepatopatias alcoólicas e hepatites virais. Estes dados encontram apoio no estudo de demais autores. ${ }^{9}$

A faixa etária predominante foi a de 40 a 49 anos, com 32\% $(n=14)$, sugerindo que a história natural da doença hepática envolve um período longo, até que as diversas hepatopatias evoluam para cirrose e câncer hepático.

A sobrevida levantada ficou em $71 \%$ para um ano, um pouco menor que em outros trabal hos. ${ }^{10-14}$ Suspeitamos que isso se deve à pequena amostra estudada ( $n=25)$, o que pode causar um viés na comparação com séries maiores.

A s principais etiologias levantadas em nosso serviço foram hepatite C e doença hepática alcoólica.

A complicação mais encontrada foi infecção, como também apontam outros autores. ${ }^{9}$ Esse mesmo trabalho destaca a sepse como a mais letal das complicações infecciosas no pós-transplante hepático, como ocorreu em nosso serviço, em que a causa mais importante de óbitos foi o choque séptico, com quatro casos.

Observamos apenas um retransplante em nossa instituição, por trombose de artéria hepática. Essa complicação realmente 
se mostra um dos principais motivos de perda dos enxertos hepáticos, juntamente com o não funcionamento primário. ${ }^{14}$ Ocorreu em três (12\%) dos pacientes estudados; um deles evoluiu com insuficiência hepática, sendo submetido a retransplante, quatro meses após o transplante primário, evoluindo com óbito por falência múltipla de órgãos, nove dias após o retransplante. Os outros dois casos evoluíram com recanalização após trombose da artéria hepática; um deles foi a óbito, cinco meses após o transplante por sepse, e o outro permaneceu vivo neste um ano de pós-transplante estudado.
No estudo atual foram registrados apenas quatro casos (16\%) derejeição do órgão, englobando somente a rejeição celular aguda, sendo este um valor significativamente baixo quando comparado com outros estudos. ${ }^{15}$

\section{CONCLUSÃO}

Relatamos um serviço em evolução, que atende pacientes de todas as regiões de Santa C atarina e, inclusive, de outros estados. A lém disso, a casuística já mostra bons resultados, com perspectivas de mel hora, pois foram estudados apenas os dois primeiros anos de evolução.

\section{ABSTRACT}

Objective: The aim of this study was to show results of the first liver transplantation surgeries in the State of Santa Catarina, performed at the Santa Isabel Hospital, Blumenau city. Methods 25 transplants were performed in 24 patients in different kinds of liver disease in the end-stage from A ugust, 2002 up to J uly, 2004. It was a transversal and quantitative study that searched the medical records of the transplantation date. It was also searched the data of other hospital admissions after the transplant. It was considered those admissions occurred up to the date of the data collection (September and October, 2005). Results: The mean age of patients was 47.24. The male sex was predominant (72\%, 18 cases). A fter three months patient's survival was $87.5 \%$, and $71 \%$ after one year. The prevailing cause of the liver insufficiency was C hepatitis associated to alcoholic liver disease (24\%), and C hepatitis (alone), al so with $24 \%$ prevalence. The most common after-surgery complication was infection (17 cases). J ust one retransplantation was performed, due to hepatic artery thrombosis. Conclusion: The aim of this review was to present results of liver transplantations performed out of the major centers with a trend to present similar results as top centers worldwide.

Keywords: Liver Transplantation; Hepatic Insufficiency ; Hepatitis, Chronic; Liver Cirrhosis; Sepsis.

\section{REFERÊNCIAS}

1. PEREIRA, W. A. Manual de Transplantes de Órgãos e Tecidos. 3 ed. Rio de Janeiro: Guanabara Koogan, 2004 - cap 11.

2. CARITHERS, R. L. J. Liver Transplantation: AASLD Practice Guidelines. Liver Transplantation 2000;6:122-135.

3. COELHO, J. C. U. Aparelho Digestivo: Clínica e Cirurgia. 2 ed. Rio de Janeiro: MEDSI, 1996. 2V - cap 108.

4. SHERLOCK, S.; DOOLEY, J. Doenças do Fígado e do Sistema Biliar. 11 ed. Rio de Janeiro: Guanabara Koogan, 2004 - cap 38.

5. PESSOA, M. G.; CURY, R. A.; BARROS, M. F. A.; SETTE JUNIOR, H. Compêndio de Hepatologia. 2 ed. São Paulo: Fundação BYK, 2001 - cap 59.

6. PortariaMS/GM no 541 de 14/03/2002. Estabelece critérios para cadastramento de candidatos a receptores de fígado - doador cadáver, no Cadastro Técnico de Receptores de Fígado - “lista única” - das CNCDO’S.

7. CHILD, C. G.; TURCOTTE, J. G. Surgery and Portal Hypertension. In: CHILD, C. G., ed. The Liver and Portal Hypertension. Philadelphia: WB Saunders, 1964;50:1:1-85.

8. WIESNER, R. H.; McDIARMID S. V.; KAMATH, P. S.; EDWARDS, E. B.; MALINCHOC, M.; KREMERS, W. K. MELD and PELD: application of survival models to liver allocation. Liver Transplantation 2001;7:567-80
9. COELHO, J. C. U.; PAROLIN, M.; MATIAS, J.; FREITAS, A.; TOLAZZI, A.; FRAGOSO, M. Infecções Hospitalares Bacterianas em uma Unidade de Transplante Hepático. Jornal Brasileiro de Transplantes Vol. 7 no 4 out./dez. 2004.

10. KIM, W. R.; WIESNER, R. H.; THERNEAU, T. M. Optimal Timing of Liver Transplantation for Primary Biliary Cirrhosis. Hepatology 1998;28:33-38.

11. SCHARSCHMIDT, B. F. Human Transplantation: Analysis of Data on 540 Patients from Four Centers. Hepatology 1984;4:95S - 101S.

12. SEABERG, E. C.; BELLE, S. H.; BERINGER, K. C. Liver Transplantation in the United States from 1987 - 1998: updated results from the Pitt- UNOS liver transplant registry. Clinical Transplants 1998:17 - 37.

13. IWATSUKI, S.; STARZL, T. E.; TODO, S. Experience in 1.000 Liver Transplants Under Cyclosporine - steroid Therapy: A Surgical Report. Transplants. Proc 1988;20:498 - 504 .

14. JAIN, A.; REYES, J.; KASHYAP, R. Sobrevida a longo prazo após transplante hepático em quatro mil pacientes consecutivos em um mesmo centro. Annals Surgery 2000;232:4:490 - 500 .

15. COELHO, J.C.U.; WIEDERKEHR, J.C.; LACERDA, M.A.; CAMPOS, A.C.L. Custo do transplante hepático no Hospital das Clínicas da Universidade Federal do Paraná. Revista da Associação Médica Brasileira 1997;43(1):53-7. 